\title{
Simple Formulae for Estimating the Depth and Dimension of Some Geological Bodies From Gravity Anomalies
}

\author{
Sayyadul Arafin
}

\author{
Department of Physics, College of Science, Sultan Qaboos University, \\ P.O. Box 36, Al Khod 123, Muscat, Sultanate of Oman.
}

عالفة بسيلة لقربب عمق وأبعاد بصن الأبسلم الجيولوجية من التبلينلت الجانبية الأرضية

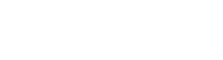

\begin{abstract}
خلاصة : قليم علاقة أوطريقة بسطة لقيلس عمق ونصف قطر بعض الأبسلم الجيولوجية البسطة مل الكرة المصمتة

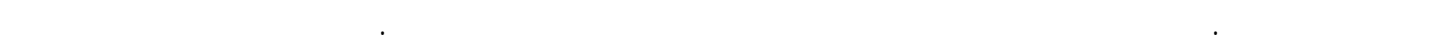

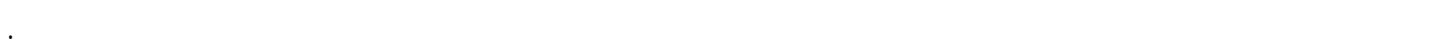

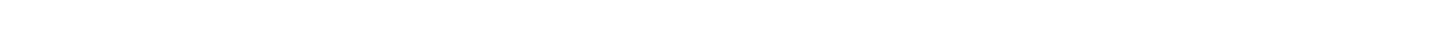

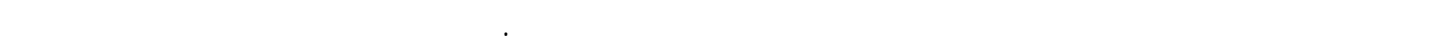

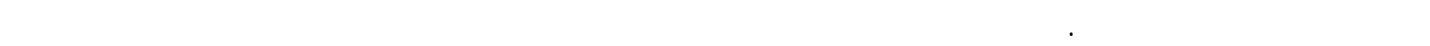

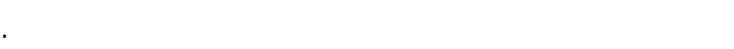

\begin{abstract}
A simple method for determining the depth and radius of some simple geological bodies such as a sphere and a horizontal cylinder is presented. The method utilizes all of the data points to calculate the body parameters. The depth is estimated from the mean of the depths determined from various fractions of the maximum anomaly and their corresponding widths. The radii of the two bodies are calculated from the areas under the respective gravity profiles. Additionally, the radial symmetry of the gravity anomaly due to a sphere has been utilized to calculate the excess mass from a single profile. The radius determined from the excess mass is independent of depth estimate. This method of determining the radius is more robust than the one based on calculating the area. An error analysis showing the effects of truncation and zero-level errors on the estimated radius is also discussed.
\end{abstract}

KEYWORDS: Gravity Anomaly, Excess Mass, Characteristic Points, Sphere and Cylinder.

\section{Introduction}

$\mathbf{E}^{2}$ arly papers on quantitative gravity interpretation found the in geophysical literature are based on the method of characteristic points. The characteristic points are the points on a gravity or magnetic profile which are easy to recognize. Such points are the maximum, minimum and inflection points ( points of maximum gradient) on the curve. Many authors including Pentz (1940), Kogbetliantz (1944), Hubert (1946), Skeels and Watson (1949), Bott and Smith (1958), Smith (1959; 1960), Milcoveanu (1970) and many others have derived formulae for geological bodies and interpreted those using characteristic curves and points. This method of interpretation is quick and useful in the field to plan future field surveys. The advent of the laptop and its capability to deal with 2-D and 3-D software (Martyn-Antienza and Garcia-Abdeslem, 1999; Ruotoistenmaki, 1992) in the field has somewhat made the method less efficient. Besides academic interest the method can still be used in the field where a laptop is either unavailable or cannot be used for lack of electricity. 


\section{SAYYADUL ARAFIN}

In the present paper a simple method of finding depth and radius of simple geological bodies such as spheres and horizontal cylinders from their gravity anomalies has been described. The method is basically similar to that described in standard geophysics text books such as Dobrin (1970) and Nettleton (1976). The present method proposes depth determination at a number of points on the profile and utilizes the area and volume (excess mass) under the gravity profile for the radius. The method has been applied on synthetic as well as field data.

\section{Method}

\subsection{Spherical Body}

The gravity anomaly caused by a spherical body (Figure 1) is given by :

$$
\Delta g(x)=\frac{4}{3} \pi G \Delta \rho R^{3}\left[z /\left(z^{2}+x^{2}\right)^{3 / 2}\right]
$$

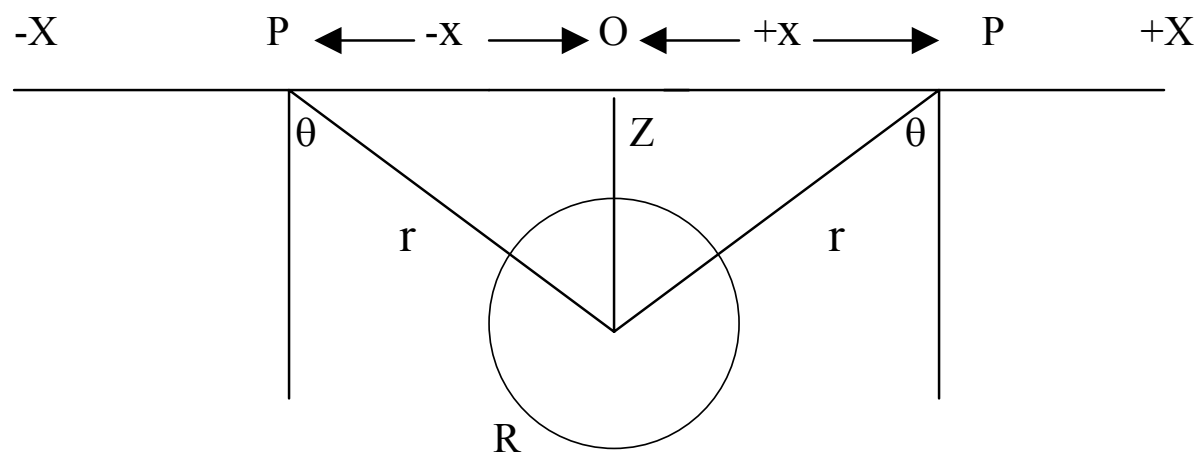

Figure 1. Symbols for spherical and horizontal bodies.

where $\Delta g(x)$ is the gravity anomaly due to the spherical body, $x$ is the horizontal distance along the gravity profile, $G$ is the universal gravitational constant, $\Delta \rho$ is the density contrast of the body with respect to the surrounding rocks, $R$ is the radius of the spherical / horizontal cylindrical body and $z$ is the depth to the center of the spherical/cylindrical body.

The anomaly $\Delta g(x)$ is symmetric about $\mathrm{x}=0$ and has its maximum amplitude at that point given by

$$
\Delta g_{\max }=\Delta g(0)=\frac{4}{3 z^{2}} \pi G \Delta \rho R^{2}
$$

The maximum value of gravity, $\Delta g_{\max }$ can be divided into $\mathrm{n}$ equal fractions giving $\mathrm{n}-1$ points on the $\mathrm{y}$ - axis. The distances on either side of $x=0$ at which these fractions occur are denoted by $x_{j / n}$, where $\mathrm{n}=2,3,4, \ldots$. and $\mathrm{j}=1,2,3, \ldots . . \mathrm{n}-1$. Index $\mathrm{j}$ here is assumed to start from the bottom part of the anomaly. The distance $2 x_{j / n}$ is the full width of the anomaly at $j / n$th fraction of the maximum amplitude. The fractions of the maximum amplitude at the corresponding half-widths $\left(x_{j / n}\right)$ are given by

$$
\Delta g\left(x_{j / n}\right)=\frac{4}{3} \pi G \Delta \rho R^{3}\left[z_{j} /\left(z_{j}{ }^{2}+x_{j / n}{ }^{2}\right)^{3 / 2}\right]=\left[\frac{4}{3} \pi G \Delta \rho R^{3} / z_{j}{ }^{2}\right] j / n
$$

It can be shown that the depth determined from the widths of the anomaly at the corresponding fractions of the maximum amplitude is given by

$$
z_{j}=x_{j / n} / \sqrt{(n / j)^{2 / 3}-1}
$$




\section{SIMPLE FORMULAE FOR ESTIMATING THE DEPTH AND DIMENSION}

It is to be noted that for $n=2$ and $j=1$, the depth $z_{1}=1.305 x_{1 / 2}$, where $x_{1 / 2}$ is the half-width of the anomaly at half of its maximum amplitude. This particular depth formula obtained is described in the books by Nettleton (1976) and Dobrin (1970) .

The depths so determined are the same for a noise free isolated anomaly. This is shown in Figures 2 and 3 for theoretical data. However, in practice the anomaly is always contaminated with noise. The estimated depth should be either the geometric or arithmetic mean of the depths, $\mathrm{z}_{\mathrm{j}}$, corresponding to different fractions of the maximum amplitude. For noisy data more depth estimates should be made within the noise free portion of the anomaly using equation (4).

Estimating the depths at different widths of the anomaly can sometimes give useful information on the shape of the body. This is explained in Example 4.

The dimension of the spherical body (i.e. the radius) can be estimated from the area under the anomaly curve. In doing so all the data points are taken into account. This is done by integrating equation (1)

$$
\int_{-\infty}^{\infty} \Delta g(x) d x=C \int_{-\infty}^{\infty}\left[z /\left(z^{2}+x^{2}\right)^{3 / 2}\right] d x
$$

where $C=4 \pi G \Delta \rho R^{3} / 3$. The left hand side of equation (5) is the area, $A$, under the gravity anomaly curve. Now changing the variable from $x$ to $\theta$ by the substitution of $x=z \tan \theta$, the limits of integration become $-\pi / 2$ and $+\pi / 2$ and the right hand side of equation (5) takes the form

$$
C \int_{-\infty}^{\infty}\left[z /\left(z^{2}+x^{2}\right)^{3 / 2}\right] d x=C / z \int_{-\pi / 2}^{\pi / 2} d \theta=2 C / z
$$

Substituting the value of the gravitational constant $G$ in the constant term $C$ and considering the distances in kilometers and kilofeet we get respectively :

$$
\begin{aligned}
\int_{-\infty}^{\infty} \Delta g(x) d x & =55.88 \Delta \rho R^{3} / z(\text { distances in } \mathrm{km}) \\
& =17.04 \Delta \rho R^{3} / z \text { (distances in kilofeet) }
\end{aligned}
$$

In reality the limits of integration are finite because our gravity profiles are finite. The left hand side of equation (6), which is the area under the observed gravity anomaly curve, can be calculated numerically by using a standard method such as the Simpson's Rule or simply by dividing the curve into very small segments and adding the area under each segment. Following the second procedure the left hand side of equation (6) can be written in digital form so that the final expression takes the form

$$
\begin{aligned}
\sum_{i=-k}^{i=k} \Delta g_{i} \Delta x_{i}= & 55.88 \Delta \rho R^{3} / z \\
& =17.04 \Delta \rho R^{3} / z
\end{aligned}
$$

$\Delta x_{i}$ 's have to be very small in order to get an accurate value of the area under the anomaly curve. It is convenient to deal with computations if the gravity values are sampled at equal intervals so that the $\Delta x_{i}$ 's can be replaced with a common value $\Delta x$. Assuming that the density contrast is known from other means of measurements, the radius $R$ now can be calculated from equation (8) or (9) depending on whether it is measured in meters or kilofeet. The calculation of $R$ in this case involves all the measured gravity values of the profile and not just a single characteristic point such as the maximum amplitude of the anomaly. The numerical value of the area under the anomaly curve is usually much larger than the maximum amplitude of the anomaly. This allows the 


\section{SAYYADUL ARAFIN}

present method to tolerate comparatively large absolute errors in the calculated value of the area. However the disadvantage of the method is that the radius depends on the estimated depth to the center of the sphere and any error in depth estimate is propagated to the estimated value of the radius. The dependence of the radius on depth is due to the fact that a sphere is a 3-dimensional body but for a horizontal cylinder the radius is independent of depth because of its two dimensionality.

\section{Radius from Excess Mass}

The gravity method is the only geophysical method that can uniquely estimate the total anomalous mass or simply the excess mass causing the anomaly. This is in fact a corollary of Gauss' flux theory in potential field theory (Parasnis, 1997). It can be shown that the excess mass can be given as the surface integral of the gravity anomaly

$$
\text { Excess } \quad \text { Mass }=K \iint \Delta g d s
$$

where $\Delta g$ 's are the gravity anomaly values measured over the surface area of the Earth and ds is an element of that surface. $K$ is a constant depending on the units used. It is $2.39 \times 10^{9}$ metric ton when area is in kilometer square and anomaly in mgals, and 26.3 imperial tons (1 imperial ton =2000lb) when area is in meter square and anomaly in mgals. (Use of non-SI units in exploration geophysics is sometimes unavoidable though unfortunate!).

The alternative method utilizes two important facts about gravity anomaly of a spherical body. Firstly the excess mass of a sphere does not depend upon its depth and secondly its gravity anomaly is radially symmetric about the centre point of the anomaly (i.e. maximum anomaly). Therefore the area of the Earth's surface on which this anomaly exists can be determined by integrating in radial coordinates. The excess mass is therefore given by

$$
\int_{0}^{\infty} \int_{0}^{2 \pi} \Delta g(r) r d r d \phi
$$

The gravity anomaly of a sphere being symmetric is independent of the radial angle $\phi$ and a function of the radial distance $r$ from the center of the anomaly. Any profile that crosses over the center point is the $x$-distance from the maximum point of the anomaly (i.e. maximum anomaly corresponds to the origin of the profile). Now substituting equation (1) in equation (11) and changing the variable from $x$ ( $r$ is actually replaced by $x$ now) to $\theta$ by the same manner as in equation (5) one gets

$$
\begin{aligned}
\text { ExcessMass } & =41.97 \times 10^{8} \Delta \rho R^{3} \text { (for distances in kilometers and gravity in milligal) } \\
& =4.62 \times \Delta \rho R^{3} \text { (for distances in meter and gravity in milligal) }
\end{aligned}
$$

The excess mass can be computed either from equation (11) in the same way used in calculating the area from equation (8) or by calculating the volume of a cone fitted to the data. The computational technique based on equation (8) has been followed in this study.

\subsection{Horizontal Cylinder}

The gravity effect of a long horizontal cylinder is the same as if its mass were concentrated on a line along its axis. The gravity anomaly of such a body at any point $x$ (Figure 1) on the horizontal surface of the earth is given by

$$
\Delta g(x)=2 \pi G \Delta \rho R^{2}\left[z /\left(z^{2}+x^{2}\right)\right]
$$

and the maximum amplitude as before occurs at $x=0$ i.e., 


$$
\Delta g_{\max }=\Delta g(0)=2 \pi G \Delta \rho R^{2} / z
$$

If we consider $n$ fractions of the maximum amplitude resulting in (n-1) number of points (Figure 3 ) denoted by $\mathrm{j}$ on the $y$-axis, then it can be shown that the depth determined from the half-widths corresponding to these points are given by :

$$
z_{j}=x_{j / n} \sqrt{[j /(n-j)]}
$$

For $n=2, j$ becomes 1 and $z_{1}=x_{1 / 2}$, the standard formula found in geophysics text books (Nettleton, 1976 ; Dobrin 1970). The radius , $R$ of the cylinder can be calculated in the same fashion as for the sphere calculated above. The final expressions for $R$ of the cylinder when the distances are measured in kilometers and kilofeet are respectively:

$$
\begin{aligned}
\sum_{i=-k}^{+k} \Delta g_{i} \Delta x_{i} & =131.66 \Delta \rho R^{2} \\
& =40.15 \Delta \rho R^{2}
\end{aligned}
$$

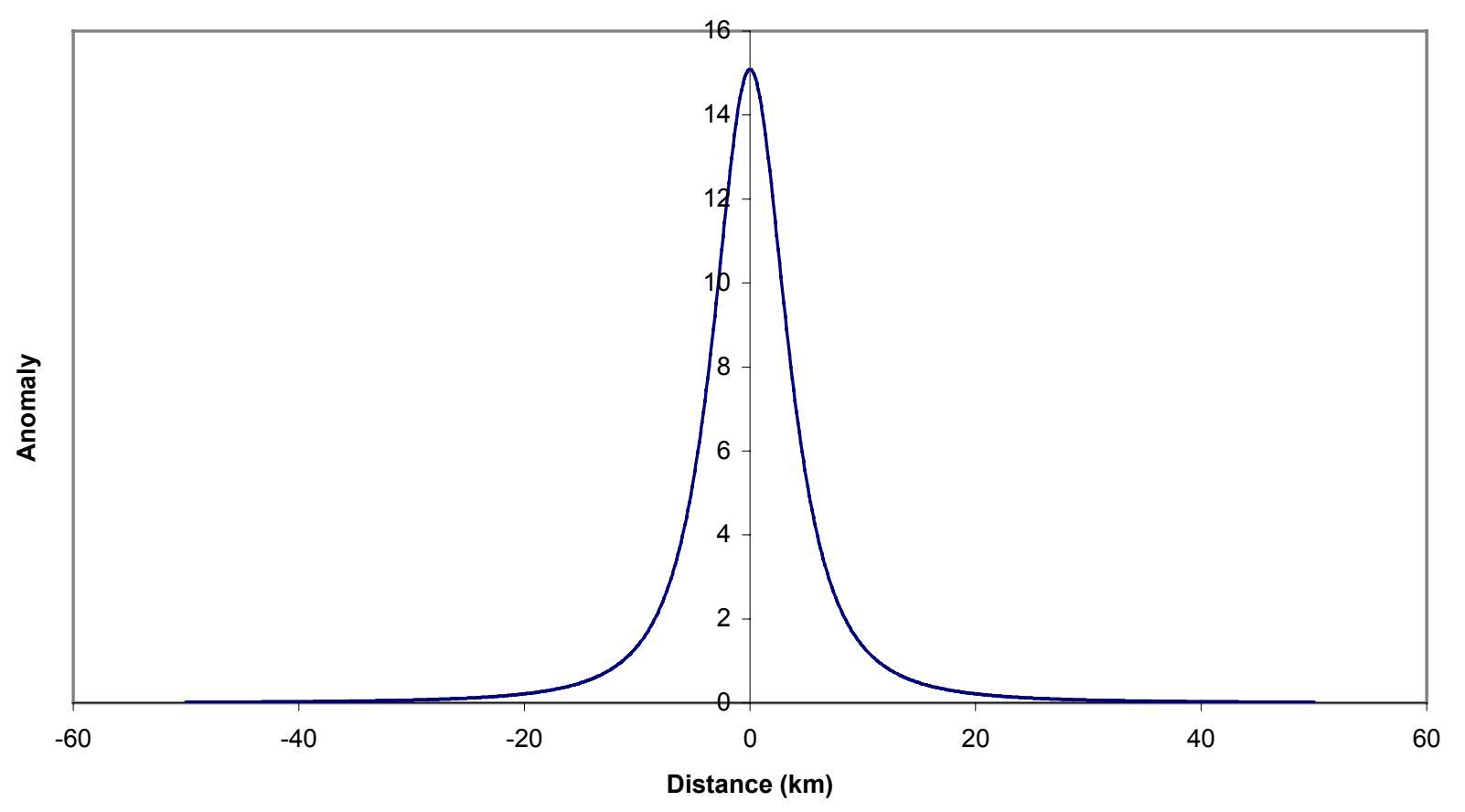

Figure 2. Interpretation of the gravity anomaly due to a sphere by the present method.

It is to be noted that the radius of the horizontal cylinder determined from the area under the curve is independent of the depth to the centre, $z$. This is unlike the method which utilizes the total amplitude to determine the radius from the formulae : $R^{2}=\Delta g_{\max } z /(41.91 \Delta \rho) \quad$ (distances in $\left.\mathrm{km}\right)$ and $R^{2}=\Delta g_{\max } z /(12.78 \Delta \rho) \quad$ (distances in $\left.\mathrm{kft}\right)$.

\section{Results and Discussions}

Four examples are presented here in order to show the validity of the present method. Two of them deal with the theoretical data and two with the field data.

\section{Example 1, Synthetic Data}

The theoretical gravity anomaly (Figure 2) for a spherical body is calculated by using equation (1). The assumed parameters of the spherical body are such that radius, $R=3.0 \mathrm{~km}$, 
depth to the center, $z=5.0 \mathrm{~km}$ and density contrast, $\Delta \rho=0.5 \mathrm{~g} \mathrm{~cm}^{-3}$. The depth and radius determined by using the present method of interpretation are $5.04 \mathrm{~km}$ and $3.00 \mathrm{~km}$, respectively. The area under the curve is $149.71 \mathrm{mgal} \mathrm{m}$.

\section{Example 2, Synthetic Data}

The theoretical anomaly and its interpretation for a horizontal cylinder are shown in Figure 3. The assumed parameters for the horizontal cylinder are the same as for the sphere. The area under the curve is $554.9 \mathrm{mgal} \mathrm{m}$. The interpreted depth is $5.06 \mathrm{~km}$ and the radius is $2.9 \mathrm{~km}$.

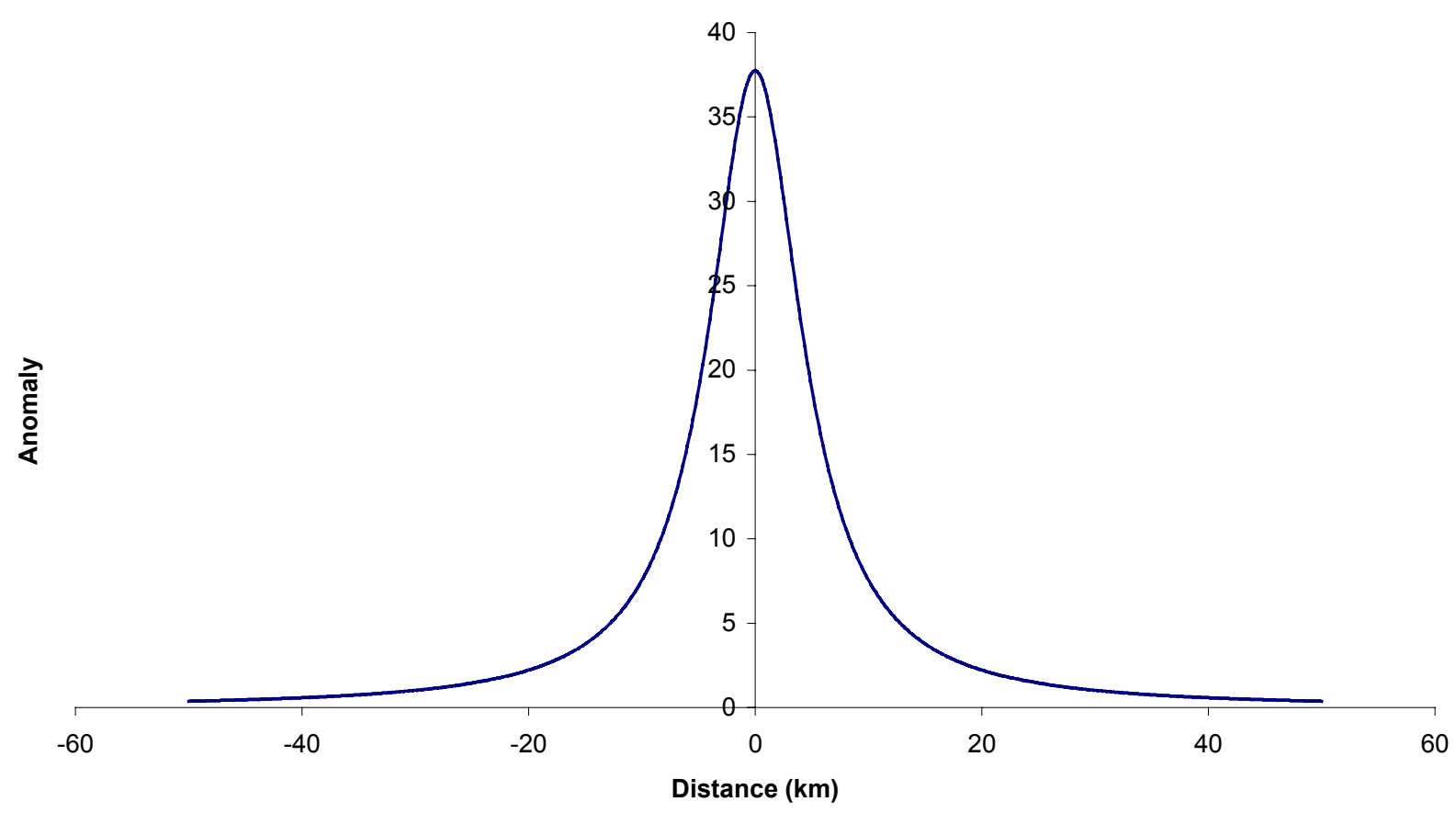

Figure 3. Interpretation of gravity anomaly due to a horizontal cylinder by the present method.

For determining the radius of the cylinder accurately the gravity values should be measured over a longer distance compared to the sphere (Figure 4). The tail of the anomaly for a horizontal cylinder continues for a much longer distance before it reduces to zero. This effect is due to contributions to gravity effects from the ends of the horizontal cylinder. Unlike the sphere , the horizontal cylinder is a two dimensional body the axial dimension of which is assumed to be infinitely long. The truncation error in the case of a horizontal cylinder is more than that of a sphere (Figure 5).

\section{Example 3, Field Data}

The method has been applied to a known geological situation, which involves anomaly due to salt dome known as Humble Salt Dome Anomaly (Nettleton, 1976).Results of interpretations of this anomaly and those of Example 4 obtained by Nettleton $(1957,1962)$ are presented in Table 1 for a comparison with those obtained from the present method. Like all salt dome anomalies this anomaly is negative indicating mass deficiency in the subsurface. The anomaly has a total amplitude of -13.9 mgal at the centre of the profile and tapers to about -0.56 mgal on each side of the centre covering a total distance of 75 kilofeet $(\mathrm{kft})$. The anomaly is quite smooth and symmetrical giving an impression of a spherical causative body. The depth determined from this anomaly by using the present method is $16.3 \mathrm{kft}$, which is the average of the depths $\left(z_{1}=15.4, z_{2}\right.$ 
$=16.1, z_{3}=16.5, z_{4}=16.6, z_{5}=16.5, z_{6}=16.7$ and $z_{7}=16.6 \mathrm{kft}$ ) determined at seven fractions of the total amplitude. The area under the anomaly curve is $407.91 \mathrm{mgal} \mathrm{kft}$ from which the radius, $R=10.92 \mathrm{kft}$, is calculated by using equation (9). The depth and radius so determined are almost the same as those ( $z=16.3$ and $R=11.3 \mathrm{kft}$ ) obtained by Nettleton. Any variation in the depths determined from the present method indicates deviation of the causative body from the assumed spherical model.

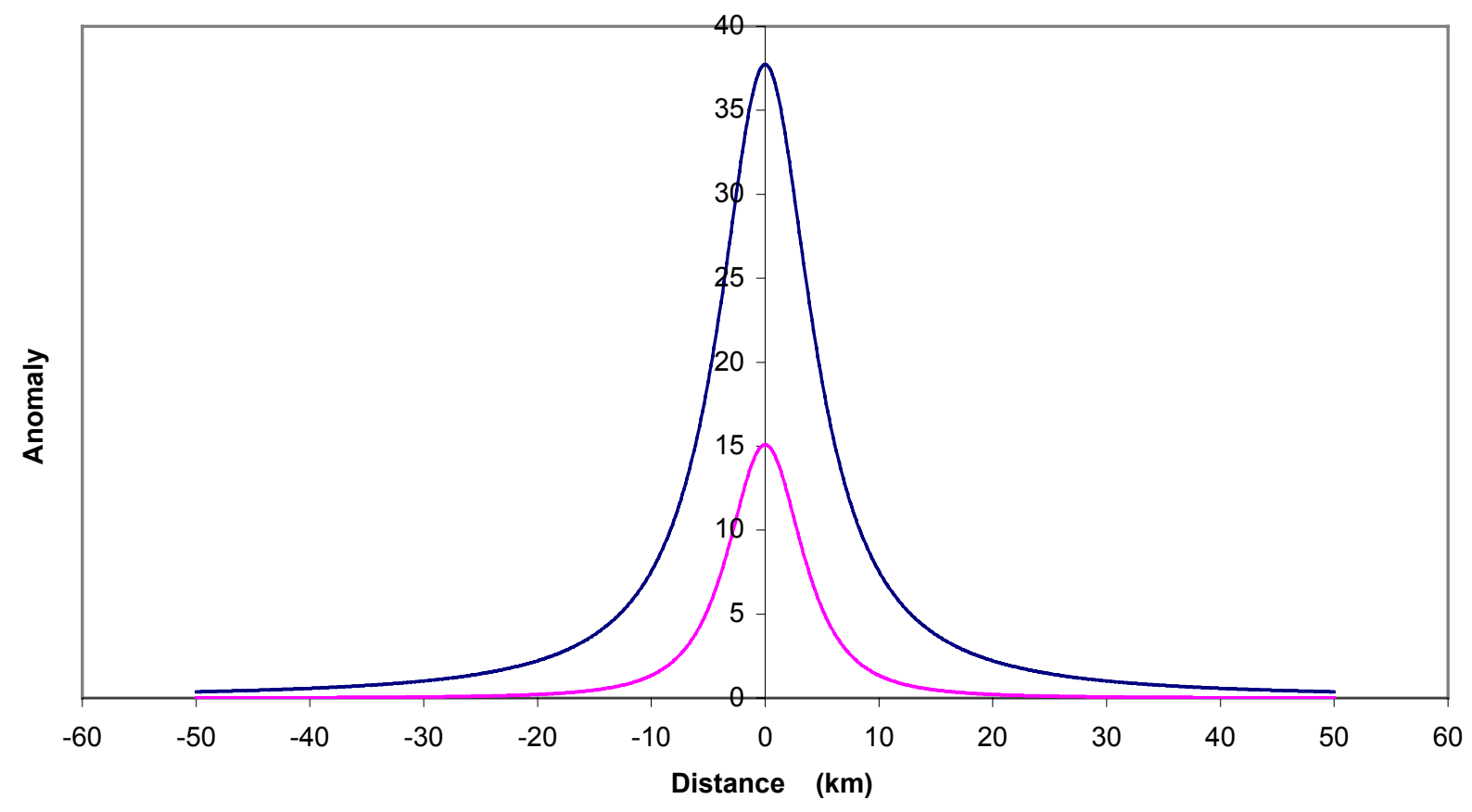

Figure 4. Comparison of profile lengths of a sphere and a horizontal cylinder.

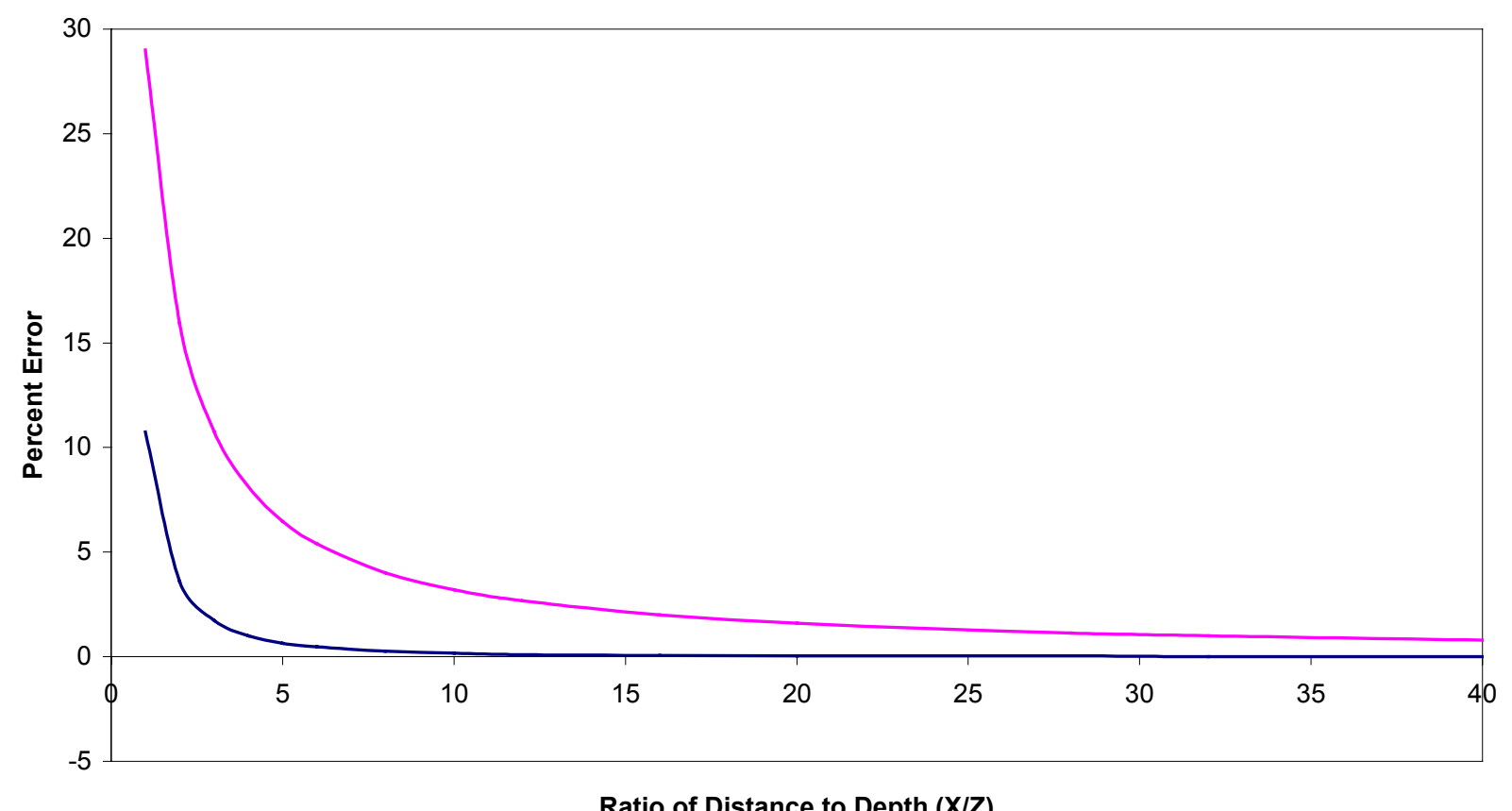

Figure 5. Truncation error on radius estimated from area under the gravity profile of a sphere and cylinder. 


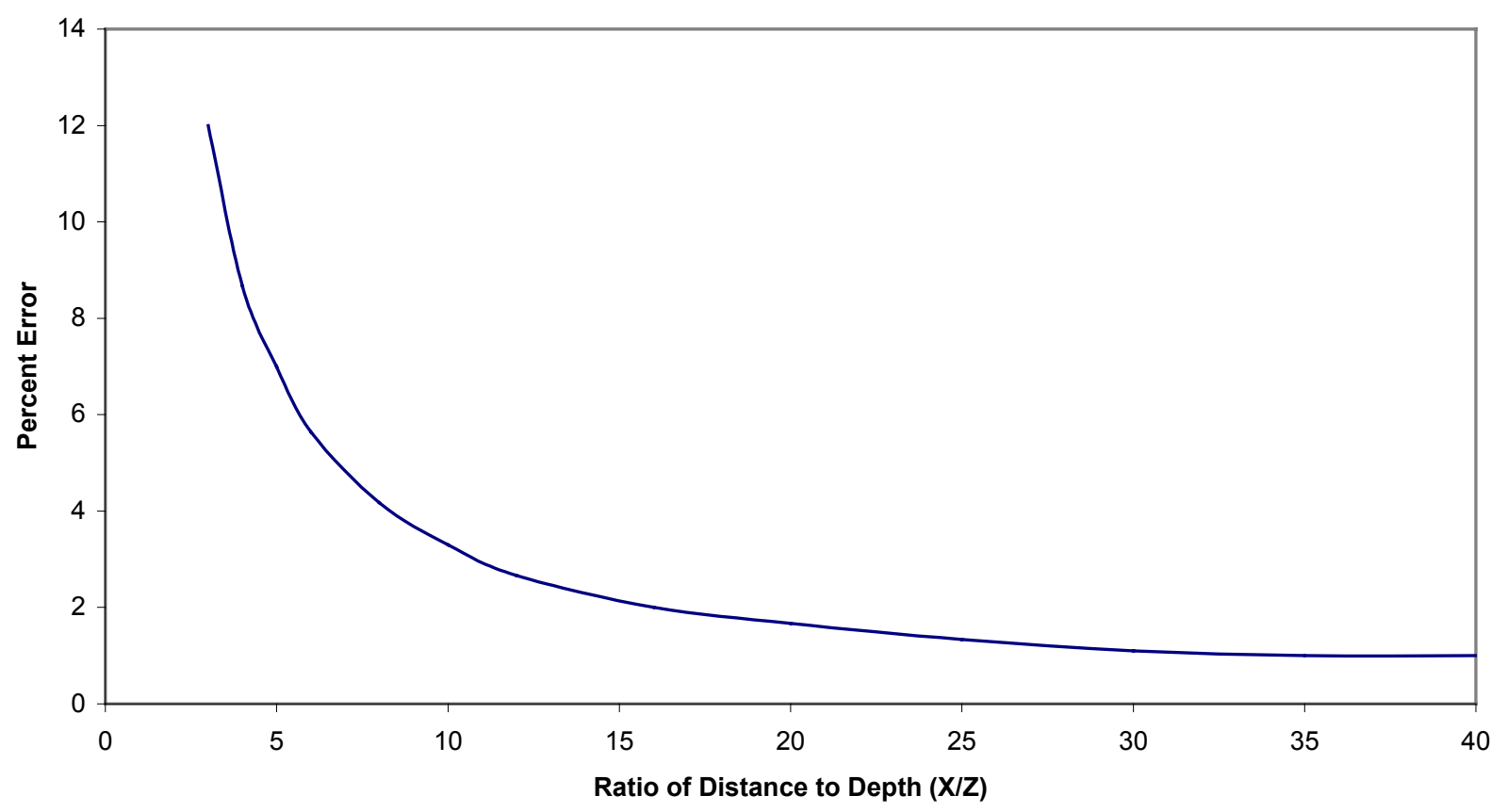

Figure 6. Truncation error on radius estimated from excess mass of a sphere.

\section{Example 4, Field data}

The more complicated Gulf of Mexico anomaly is interpreted by Nettleton (1957) by a mushroom-shaped geological body instead of a sphere. The interpretation of the same anomaly with a spherical model is not meant for a comparison but to show that estimating the depth at different fractions of the maximum amplitude can give us information on the validity of the assumed spherical model. This anomaly has a total amplitude of about $-9.0 \mathrm{mgal}$ and is distorted at the central part of the anomaly making the determination of the total amplitude a subjective matter. The distortion at the centre of the anomaly is believed to be due to higher density of the shallow uppermost part of the dome relative to the density of the surrounding sediments.

Table 1: Parameters of the geological bodies obtained by Nettleton (1957) and the present method.

\begin{tabular}{|c|c|c|}
\hline Anomalies & Nettleton & Present Method \\
\hline Humble Salt Dome, Example 2 & $R=11.3 \mathrm{kft} ; z=16.3 \mathrm{kft}$ & $R=10.92 \mathrm{kft} ; z=16.3 \mathrm{kft}$ \\
\hline Gulf of Mexico, Example 3 & $\begin{array}{c}\text { Mushroom-shaped body } \\
\text { Top radius : } 15 \mathrm{kft}, \\
\text { Bottom radius } 7.5 \mathrm{kft}\end{array}$ & $\begin{array}{c}\text { is not determined } \\
z=22.4 \mathrm{kft}\end{array}$ \\
\hline
\end{tabular}

This positive density contrast produces a positive anomaly at the centre which reduces the peak amplitude of the anomaly. Nettleton has interpolated the observed amplitude to about -10.0 mgal in his interpretation of the anomaly in terms of a mushroom-shaped body the top of which has positive density contrast. The maximum horizontal dimension of the mushroom is about $30,000 \mathrm{ft}$ at the top , minimum about $15,000 \mathrm{ft}$ at the bottom and the depth to the top of the dome is about $1,300 \mathrm{ft}$. Depth estimates $\left(z_{1}=21.2, z_{2}=20.1, z_{3}=21.9, z_{4}=23.1, z_{5}=24.4, z_{6}=24.9\right.$ and $z_{7}$ $=26.1 \mathrm{kft}$ ) for this anomaly show a wide range of values indicating that the causative body is far from being spherical in shape. $z_{1}$ is the depth determined for the half-width closest to the minimum amplitude (i.e. bottom part of the anomaly) and $z_{7}$ for the half-width closest to the 
maximum amplitude ( i.e. top part of the anomaly). Depth determination from equation (4) depends upon the half-width of the anomaly. The gradual but fairly large increase in depths as one estimates depths from bottom to top of the anomaly indicates that the widths towards the top of the anomaly are increasing more than expected. This in turn means that the causative body is widening towards its top shaping up something like an inverted pyramid.

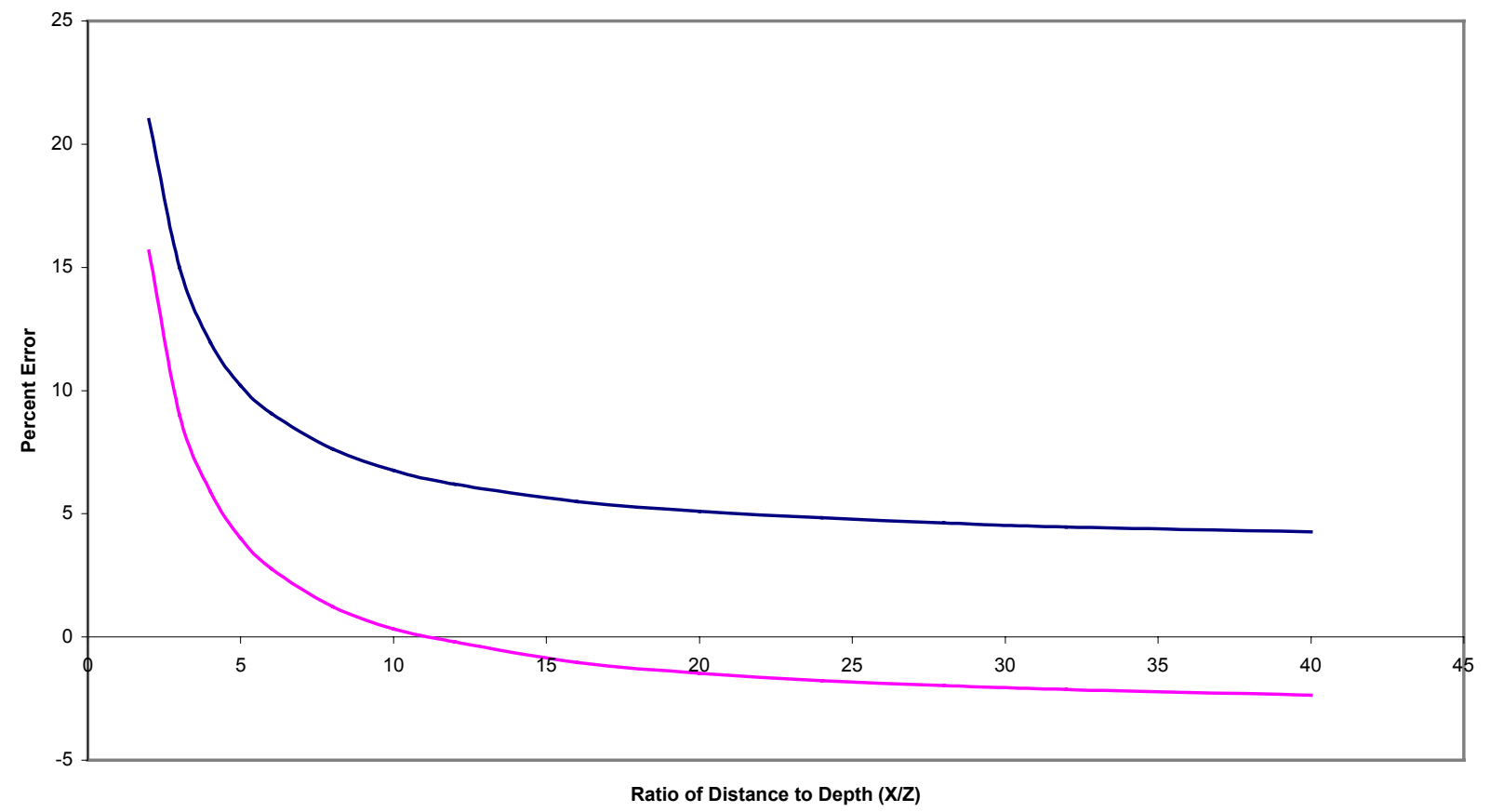

Figure 7. Combined truncation and zero-level error on radius estimated from excess mass of a sphere.

There are two possible sources of error that will influence the radius estimate. The truncation error arises because of the finite length of the gravity profiles. Therefore the computed areas under the gravity profiles of a sphere and a cylinder and the computed excess mass of a sphere will always be less than what would have been obtained from a semi-infinite profile length. As a result the estimated value of the radius will be less than the actual value. The affects of this error are shown in Figure 5 and Figure 6. It can be seen from Figure 5 that the truncation error is more for a cylinder than a sphere. For example for a $10 \%$ error in the radius estimate, the minimum $x / z$ value is 1 in the case of a sphere and about 3.3 in the case of a cylinder. For the same error the profile length, in the case of radius estimate from the excess mass of a sphere, has to be about 3.5 times more than the profile length in the case of area estimate.

The zero-level error is introduced when the regional gravity values are not properly removed from the data. The incorrect removal of the regional will place zero-level of the anomaly (i.e. $x$ axis ) either above or below the true level thus either decreasing or increasing the true gravity values respectively. The combined affect of the truncation error and the zero-level error is shown in the case of excess mass estimate for a sphere in Figure 7. The curve above is obtained by under-subtracting the regional by $10 \%$ and the curve below by over-subtracting by the same amount.

\section{Conclusion}

Depth to the center of geological bodies of simple geometrical shapes such as a sphere and a cylinder has been determined at a number of points along the gravity profile. The radius of the 
bodies is obtained from the area under the gravity profile. The radius so determined depends on the depth estimate in the case of a sphere. An alternative method based on calculating excess mass from the gravity anomaly of a sphere determines the radius which is independent of the estimated depth. A single gravity profile for a sphere has been used to calculate the excess mass by utilising the radial symmetry of such a profile. Depth determination at different parts of the anomaly can give us information on the validity of the assumed spherical shape of the body. Since the numerical values of the area under the gravity profile and the excess mass are much larger than the total amplitude of the anomaly the radius determined from these values is expected to be more tolerant to error than the radius estimated from a single point such as a maximum or inflection point in the profile. However this is not valid in the case of a horizontal cylinder because the truncation error is more dominant in this case resulting in less accurate estimate of the area under the observed gravity profile. The method of radius estimate from the excess mass is more robust than the method that uses the area.

\section{References}

BOTT, M.H.P.and SMITH, R.A. 1958. The estimation of the limiting depth of gravitating bodies, Geophysics. $6: 1-10$.

DOBRIN, M.B. 1970. Introduction to Geophysical Prospecting ( ${ }^{\text {rd }}$ Edition), McGraw - Hill Publishing Co., New York.

HUBERT, M.K. 1946. A line integral method of computing the gravimetric effects of 2-D bodies. Geophysics. 13: 215-221.

KOGBETLIANTZ, E.G. 1944. Quantitative Interpretation of magnetic and gravity Anomalies. Geophysics. 9: 463-473.

MILCOVEANU, D.1970. Some formulas useful in the interpretation of gravitational and magnetic profiles. Geophysics. 35: 66-73.

MARTYN-ANTIENZA, B. and GARCIA-ABDESLEM, J. 1999. 2-D gravity modeling with analytically defined geometry and quadratic polynomial density functions, Geophysics. 64: 1730-1734.

NETTLETON, L.L.1976. Gravity and Magnetics in Oil Prospecting (2 ${ }^{\text {nd }}$ Edition), McGraw-Hill Publishing Co., New York.

NETTLETON, L.L.1962. Gravity and magnetics for Geologists and Seismologists, Bulletin American Association of Petroleum Geologist. 46: 1815-1838.

NETTLETON, L.L.1957. Gravity Survey over a Gulf Coast Continental Mound, Geophysics. 22 : 630-642

PARASNIS, D.S., 1997. Principles of Applied geophysics. Chapman and Hall, London.

PENTZ, H.H., 1940. Formulas and curves for the interpretation of certain 2-D magnetic and gravity anomalies. Geophysics. 5: 295-306.

RUOITSTENMAKI, T., 1992. The gravity anomaly of two dimensional sources with continuous density distribution and bounded by continuous surfaces, Geophysics. 57: 623-628

SKEELS, D.C. and WATSON, R.J. 1949. Derivation of magnetic and gravimetric quantities by surface integrals. Geophysics. 14: 133-145.

SMITH, R.A., 1960. Some formulae for interpreting local gravity anomalies. Geophysics. 8 : 607 613.

SMITH, R.A., 1959. Some depth formulae for local magnetic and gravity anomalies. Geophysics. 7: 55-63. 